\section{RESEARCH PERSPECTIVES}

\author{
D.A. Brown \\ A.N. Hasso
}

\section{Toward a Uniform Policy for Handling Incidental Findings in Neuroimaging Research}

\begin{abstract}
SUMmARY: A recent study carried out at the University of California, Irvine exemplifies a commonly overlooked ethical conundrum of neuroimaging research: incidental findings. Research study designs must address the potential of uncovering unexpected findings in subjects during the study and delineate a protocol for reporting and initiating treatment. We urge the community to petition their home institutional review board to mandate inclusion of an incidental findings protocol into all neuroimaging research applications.
\end{abstract}

$\mathbf{T}$ he venerated rocket scientist Werner von Braun was once quoted as saying, "Research is what I'm doing when I don't know what I'm doing." Although history assures us that Dr. von Braun at least occasionally knew what he was doing, his words are as relevant to brain exploration today as they were to space exploration 60 years ago. Science is inherently uncertain, and any seasoned scientist would agree that research is as much of a venture into the unknown as it is a succession of failures. Nevertheless, the exploration of any frontier carries implicit responsibility for the explorer. Neuroimaging has enjoyed astonishing advances in its ability to generate information on the human brain. Advances in the doctrine of moral responsibility for this information, however, have not always been as forthcoming.

A recent study carried out by the Department of Psychiatry and Human Behavior at the University of California, Irvine exemplifies a commonly overlooked ethical conundrum of neuroimaging research-that of incidental findings. In an effort to study the influence of prenatal steroids on childhood brain development, a neuroscience research group requested our involvement in evaluating the MR imaging brain scans of 150 children. Although the protocol for measuring the volumes of the hippocampus and amygdala as well as the associated subregions of the brain was meticulously defined in the institutional review board (IRB) application, no mention was made of the possibility of uncovering pathologic findings outside the expected parameters of the study. The application was careful to substantiate the scientific responsibility of the study, but fewer details were provided on the issue of clinical responsibility. In particular, who is the experienced team member who would supervise the follow-up care of these children if something was found? Who would provide the care? Are the radiologists reading the films responsible for missed findings? The study proceeded, and 150 apparently health children were whisked through head MR imaging to probe their brains at the deepest and darkest level, with the comfortable expectation that we would measure various volumes and densities in otherwise unremarkable films.

The story that follows should not be surprising. Incidental

\section{Received and accepted May 27, 2008}

From the School of Medicine (D.A.B.), University of California, Irvine, Orange, Calif; and Department of Radiological Sciences (A.N.H.), University of California, Irvine Medical Center, Orange, Calif.

Please address correspondence to Anton N. Hasso, MD, FACR, Department of Radiological Sciences, University of California, Irvine Medical Center, 101 City Dr S, Orange, CA 92668-3298; e-mail: anhasso@uci.edu

DOI 10.3174/ajnr.A1227 brain lesions that were not previously known turned up in the MR imaging studies of 2 children. A small cyst adjacent to the left frontal horn with associated ventriculomegaly and hemispheric volume loss was seen in 1 subject, along with a hypoplastic brain stem. Such findings are thought to be due to an ischemic perinatal insult, which could correlate with developmental delays. In another subject, a complex congenital malformation was found, evidenced by focal right frontal cortical pachygyria and focal heterotopia medially near the right frontal horn, with midline morphologic dysplasias, including a thinned corpus callosum and an absent septum pellucidum. With these findings and through no conscious decision of anyone involved, an innocent research project had taken an unanticipated turn. Whether prenatal steroids influence brain development was no longer important for these children and their families; the issue had become whether the children now required treatment for their newly discovered brain abnormalities and what this might portend for their futures.

\section{Prevalence of Incidental Findings}

There is little doubt that the unexpected lies in the unknown. The prevalence of recognizable and clinically significant neuropathologies is thought to fall between $2 \%$ and $8 \%$ in the general population, and deviations from normal may occur in $\leq 20 \%$ of individuals. ${ }^{1-6}$ The significance of these findings may be profound. An MR imaging study of 2536 healthy young men applying for flying duties in the German Air Force revealed arachnoid cysts in $1.7 \%$, vascular abnormalities in $0.51 \%$, and intracranial tumors in $0.47 \%$ of the men. ${ }^{6}$ Similarly, a retrospective study of asymptomatic volunteers in the United States demonstrated primary brain tumors in $0.2 \%$ of a diverse study population. ${ }^{3}$ A recently published study of the 2000 people in the Rotterdam study population (mean age, 63.3 years) with high-resolution structural brain MR imaging (1.5T) demonstrated asymptomatic brain infarcts in $7.2 \%$, cerebral aneurysms in $1.8 \%$, and primary brain tumors in $1.6 \%$ of the patients. ${ }^{5}$ The question for the neuroimaging researcher is not whether asymptomatic pathologies exist in their study population, but rather how many will be found.

In terms of clinical attention, the need for follow-up and referral will depend on the study population, but planning should always begin with the assumption that the rate will be nonzero. For example, a retrospective study published in 2004 showed that $6.6 \%$ of healthy volunteers required referrals on the basis of incidental findings from brain MR imaging. ${ }^{8}$ In higher risk populations, these odds can be considerably greater: Fully $84 \%$ of volunteers with past lead exposure had inci- 
dental findings on brain MR imaging, of whom $42 \%$ required routine referral, $14 \%$ required urgent referral, and $1.3 \%$, immediate referral. ${ }^{1}$

Equivalent results have been described in pediatric populations. In a retrospective study of 225 healthy children, aged 1 month to 18 years, incidental findings were detected in $21 \%$ of volunteers, of whom $7.7 \%$ required referral. ${ }^{9}$ Likewise, incidental findings were detected in $25.7 \%$ of 666 patients at a pediatric neurology practice, of which $17 \%$ were considered normal variants and $8.7 \%$, abnormal; and $0.3 \%$ required a neurosurgery referral. ${ }^{2}$ Taken together, these studies underscore the need for realistic referral guidelines built into any neuroimaging research protocol, despite the apparent salubrity of the subjects.

A question that naturally arises is on whom the responsibility for treatment should fall. The principal investigator (PI), though presumably responsible for the on-site safety and informed consent of the subjects, will not necessarily be trained in image interpretation. If the scans are passed on to someone who is trained, does that individual become responsible for informing the subject and coordinating follow-up care? Does that specialist become liable for missed findings? Should it be mandatory to have all research brain scans read by a boardcertified neuroradiologist with a certificate of added qualification (CAQ)? The ethical-legal web becomes tangled indeed.

\section{Research Practices}

Lawful responsibilities notwithstanding, volunteers for neuroimaging research studies may have inherent expectations about what information will be obtained and shared. In 1 study, questionnaires given to 105 healthy people who previously participated as control subjects in neuroimaging research revealed that the overwhelming majority (97\%) expected that abnormal findings would be communicated to them, despite the significance. ${ }^{9}$ A slight majority (59\%) expected that these findings would be communicated to them by a physician affiliated with the research team.

Unfortunately, there is a discontinuity when comparing these expectations with current research practices. An on-line survey of 74 neuroimaging researchers revealed that $82 \%$ of the respondents found incidental abnormalities such as arteriovenous malformations, brain tumors, and developmental abnormalities in their studies. ${ }^{7}$ The protocols for handling such findings varied widely. Thirty-six percent reported that scans are read by a neuroradiologist and all findings are disclosed, $47 \%$ reported that only suggestive findings are disclosed, $4 \%$ responded that disclosure depended on the type of study, and 13\% responded that incidental findings were not disclosed at all.

Should investigators report all atypical findings in all neuroimaging research studies to the subject? Significant findings, benign or otherwise, can have a profound medical and psychological impact on the individual. Some argue that a high rate of false-positive findings may exist, ${ }^{10}$ which further complicates the question. There are well-recognized ethical arguments in the genetics literature supporting a subject's right not to know, ${ }^{11}$ and in many cases, paranoia of the potential consequences would truly impact the quality of life of these patients. Could disclosure of an underlying condition precipitate the onset of symptoms as a somatization effect? Have we truly done no harm by revealing this information to our patient?

\section{Bethesda Conference}

The problem of incidental findings has not gone unnoticed among neuroscientists. ${ }^{12}$ In recent years, Judy Illes, a Stanford University neuroimaging researcher and bioethicist, is among those leading the push to define a set of guidelines for reporting incidental findings. A January 2005 workshop in Bethesda, Md, sponsored by the National Institutes of Health (NIH) and Stanford University, convened 40 distinguished scientists and scholars from the United States and Canada to address the issue of incidental findings in neuroimaging research. ${ }^{13}$ Illes published a summary of the conference in Science, highlighting the major topics of discussion among the participants. ${ }^{10}$

One of the working groups of the Bethesda conference discussed issues surrounding the detection of incidental findings. It was agreed that the imaging methods of a research proposal should be the minimum required to address the aims of the study. Subject selection should follow sound scientific rationale, dictated by the aims of the research, and subjects should not be excluded on the basis of the likelihood of detecting incidental findings. Scanning should be performed by a competent technician, examined by a qualified researcher, and read by a radiologist in a timely manner if consultation is deemed necessary by the PI. A majority of the group agreed that a research protocol that provides for disclosure of suggestive incidental findings to the subjects is "ethically desirable." However, because of the potential for false-positive findings, some members of the working group believed that it was improper to communicate all but the most certain findings.

The issue of responsibility of care played a prominent role at the Bethesda conference. The conclusion among the participants was that the subject's primary care provider (PCP) should have the ultimate responsibility of assessment of the findings and coordination of follow-up care, provided that the radiologist communicates these issues to the PI and the PI communicates them to the PCP. A consensus was not reached regarding the issue of whether a board-certified radiologist, CAQ neuroradiologist, physician competent in reading neuroimaging scans, or other professional should be required to read the images. Most of the working group did not favor this requirement.

The conference also concluded that the IRB at the research site must assume a primary role in enforcing the inclusion of an incidental findings protocol in human research proposals. The method for handling incidental findings should be explicitly defined in the research proposal and in the written and verbal informed consent process with the subjects. Thus, 2 conduits of communication regarding the potential for incidental findings are essential when obtaining consent-from the PI to the IRB and from the PI to the research subjects.

\section{Future Directions}

The field of radiology is coming to a crossroads, where the issue of responsibility of care will guide many future directions for the field. In recent years, a number of precedents for radiology practice have been fixed without great input from radiologists. The Mammography Quality Standards Act (MQSA) of 1992 is 1 example of such precedents. In response to the 
realization by women's advocates, radiologists, and legislators that mammography quality was quite variable, a number of rigorous standards for mammography interpretation were written into law. The composite effect of MQSA accreditation has undoubtedly resulted in improved mammographic standards, with $18 \%$ more facilities passing the accreditation test on the first attempt in 2003 than in the period from 1987 to 1991. ${ }^{14}$ However, with the MQSA mandate that "a summary of the written report shall be sent directly to the patient in terms easily understood by a lay person," the role of the radiologist is drawn more toward direct responsibility for the patient and farther from that as a consultant. ${ }^{14}$ Despite its clinical success, some have commented that MQSA has increased the bureaucratic hassles with which radiologists must deal. ${ }^{15}$ Will the responsibility for incidental findings also come to rest on the radiologist? The answer is unclear.

The power and sensitivity of neuroimaging present a double-edged sword. Scanning the brain will inevitably uncover anomalies and pathologies, and it is the duty of researchers to ensure that a subject-centered approach is followed in the management of these findings. Although much discussion on this topic has transpired, a standard protocol for detection and disclosure of incidental findings has not yet been widely implemented. Even the NIH does not yet require inclusion of an incidental findings protocol into grant applications, though certain guidelines are suggested. ${ }^{16}$

We believe that the ethical issues surrounding the detection and disclosure of incidental findings are best addressed in the research study design. An ethically sound neuroimaging research study must address the possible-if not probableevent of uncovering unexpected potentially pathologic findings in subjects during the study and must delineate a protocol for reporting and initiating treatment for these findings. The IRB or equivalent oversight body at the research center is critical to enforcing these guidelines and should consider the handling of incidental findings by the study when granting approval. Until a uniform national policy is implemented, we urge the neuroimaging community to petition their home IRB to mandate inclusion of an incidental findings protocol into all neuroimaging research applications. Clarifying the procedure for unexpected findings will benefit the PI, the radiologist, and most important, the subject.
Neuroimaging affords a precious and personal insight into human beings, but beneficence demands that any relevant information be shared with the subject and that his or her welfare take first priority. The excitement of exploration will always serve as motivation for the progression of science, but we must reserve the simplicity of Dr. von Braun's words for simpler endeavors. Even if we don't know what we're doing in brain research, we can at least know what we will do for our subjects.

\section{References}

1. Alphs HH, Schwartz BS, Stewart WF, et al. Findings on brain MRI from research studies of occupational exposure to known neurotoxicants. AJR Am J Roentgenol 2006;187:1043-47

2. Gupta SN, Belay B. Intracranial incidental findings on brain MR images in a pediatric neurology practice: a retrospective study. J Neurol Sci 2008;264:34-37

3. Katzman GL, Dagher AP, Patronas NJ. Incidental findings on brain magnetic resonance imaging from 1000 asymptomatic volunteers. JAMA 1999;282:36-39

4. Kim BS, Illes J, Kaplan RT, et al. Incidental findings on pediatric MR images of the brain. AJNR Am J Neuroradiol 2002;23:1674-77

5. Vernooij MW, Ikram MA, Tanghe HL, et al. Incidental findings on brain MRI in the general population. New Engl J Med 2007;357:1821-28

6. Weber $\mathrm{F}$, Knopf $\mathrm{H}$. Incidental findings in magnetic resonance imaging of the brains of healthy young men. J Neurol Sci 2006;240:81-84

7. Illes J, Kirschen MP, Karetsky K, et al. Discovery and disclosure of incidental findings in neuroimaging research. J Magn Reson Imaging 2004;20:743-47

8. Illes J, Rosen AC, Huang L, et al. Ethical consideration of incidental findings on adult brain MRI in research. Neurology 2004;62:888-90

9. Kirschen MP, Jaworska A, Illes J. Subjects' expectations in neuroimaging research. J Magn Reson Imaging 2006;23:205-09

10. Illes J, Kirschen MP, Edwards E, et al. Ethics: incidental findings in brain imaging research. Science 2006;311:783-84

11. Wilson J. To know or not to know? Genetic ignorance, autonomy, and paternalism. Bioethics 2005;19:492-504

12. Illes J, Kirschen MP, Edwards E, et al. Practical approaches to incidental findings in brain imaging research. Neurology 2008;70:384-90

13. Detection and disclosure of incidental findings in neuroimaging research $(\mathrm{NIH}$ and Stanford University). January 6-7, 2005; Bethesda, Md; Available at: http:// www.ninds.nih.gov/news_and_events/proceedings/ifexecsummary.htm. Accessed June 19, 2008

14. Destouet JM, Bassett LW, Yaffe MJ, et al. The ACR's Mammography Accreditation Program: ten years of experience since MQSA. J Am Coll Radiol 2005;2:585-94

15. Levin DC, Rao VM, Bree RL, et al. Turf battles in radiology: how the radiology community can collectively respond to the challenge. Radiology 1999;211:301-05

16. Frequently asked questions from applicants: human subjects protectionsinformed consent, incidental findings, resolving concerns. NIH office of extramural research. Available at: http://grants.nih.gov/grants/policy/hs/faqs_aps_ hsp.htm. Accessed March 22, 2008 